\title{
AUTOMORPHISMS OF COMPACT NON-ORIENTABLE RIEMANN SURFACES
}

\author{
by D. SINGERMAN
}

(Received 1 August, 1969)

1. Introduction. Using the definition of a Riemann surface, as given for example by Ahlfors and Sario, one can prove that all Riemann surfaces are orientable. However by modifying their definition one can obtain structures on non-orientable surfaces. In fact nonorientable Riemann surfaces have been considered by Klein and Teichmüller amongst others. The problem we consider here is to look for the largest possible groups of automorphisms of compact non-orientable Riemann surfaces and we find that this throws light on the corresponding problem for orientable Riemann surfaces, which was first considered by Hurwitz [1]. He showed that the order of a group of automorphisms of a compact orientable Riemann surface of genus $g$ cannot be bigger than $84(g-1)$. This bound he knew to be attained because Klein had exhibited a surface of genus 3 which admitted $P S L(2,7)$ as its automorphism group, and the order of $P S L(2,7)$ is $168=84(3-1)$. More recently Macbeath $[5,3]$ and Lehner and Newman [2] have found infinite families of compact orientable surfaces for which the Hurwitz bound is attained, and in this paper we shall exhibit some new families.

2. Riemann surfaces and NEC groups. By a Riemann surface in this paper we shall mean a surface $S$ together with an open covering by a family of sets $\mathscr{U}=\left\{U_{i}\right\}$ with the properties plane.

1. For each $U_{i} \in \mathscr{U}$ there exists a homeomorphism $\phi_{i}: U_{i} \rightarrow \mathrm{C}$, where $\mathbf{C}$ is the complex

2. If $U_{i}, U_{j} \in \mathscr{U}$ and $U_{i} \cap U_{j} \neq \emptyset$ then $\phi_{i} \circ \phi_{j}^{-1}$ is a conformal or anticonformal mapping defined on $\phi_{j}\left(U_{i} \cap U_{j}\right)$.

A homeomorphism $f: S \rightarrow S$ is called an automorphism if $\phi_{i} \circ f \circ \phi_{j}^{-1}$ is either a conformal or anticonformal mapping in its domain of definition. If $S$ is an orientable surface and $f$ is orientation preserving (reversing) then $f$ is called a +automorphism (-automorphism). (In the papers of Macbeath, Lehner and Newman etc., their automorphisms are +automorphisms.)

+ Automorphisms of orientable Riemann surfaces have been studied by means of Fuchsian groups. We shall study automorphisms of non-orientable Riemann surfaces by means of the non-Euclidean crystallographic (NEC) groups introduced by Wilkie [7].

Let $D$ denote the upper-half complex plane and $\mathscr{G}$ the group of conformal and anticonformal homeomorphisms of $D$. The elements of $\mathscr{G}$ are the transformations of the form

(i) $z \rightarrow \frac{a z+b}{c z+d} \quad a, b, c, d$ real, $a d-b c=1$,
(ii) $z \rightarrow \frac{a \bar{z}+b}{c \bar{z}+d} \quad a, b, c, d$ real, $a d-b c=-1$.

The elements of type (i), the conformal homeomorphisms, form a subgroup of index 2 in $\mathscr{G}$ which we denote by $\mathscr{G}^{+}$. 
$\mathscr{G}$ has a topology induced by the numbers $a, b, c, d$. It has two components, namely $\mathscr{G}^{+}$ and $\mathscr{G}-\mathscr{G}^{+}$. An NEC group is a discrete subgroup of $\mathscr{G}$ and an NEC group contained in $\mathscr{G}^{+}$ is called a Fuchsian group. An NEC group which contains elements of $\mathscr{G}-\mathscr{G}^{+}$, i.e. orientation reversing elements, will be called a proper NEC group. We shall only be interested in NEC groups $\Gamma$ for which the orbit space $D / \Gamma$ is compact, and in this paper the term NEC group means a discrete subgroup of $\mathscr{G}$ with compact orbit space. Similarly by a surface we shall mean a compact surface.

Associated with an NEC group $\Gamma$ we have a $\Gamma$-fundamental region which is defined in exactly the same way as for Fuchsian groups. Moreover many of the basic results concerning the fundamental region are the same for NEC groups as for Fuchsian groups. For example the non-Euclidean area of a fundamental region of an NEC group depends only on the group and not on the region chosen. Thus we can denote the non-Euclidean area of a fundamental region for $\Gamma$ by $\mu(\Gamma)$. Then if $\Lambda$ is a subgroup of finite index in $\Gamma$ we have

$$
|\Gamma: \Lambda|=\frac{\mu(\Lambda)}{\mu(\Gamma)}
$$

In particular, if $\Gamma$ is a proper NEC group, then it has a subgroup $\Gamma^{+}$of index 2 consisting of the elements which preserve orientation (i.e. $\Gamma^{+}=\Gamma \cap G^{+}$). $\Gamma^{+}$is called the canonical Fuchsian group of $\Gamma$, and we have, from (1),

$$
\mu\left(\Gamma^{+}\right)=2 \mu(\Gamma)
$$

From a $\Gamma$-fundamental region Wilkie obtained a presentation for $\Gamma$. From his work we deduce that there are two classes of groups which act without fixed points. The first type, which we denote by $\Lambda_{g}$, has presentation

$$
\left\{a_{1}, b_{1}, \ldots a_{g}, b_{g} ; \prod_{i=1}^{\theta}\left[a_{i}, b_{i}\right]=1\right\} \quad(g \geqq 2),
$$

where $\left[a_{i}, b_{i}\right]=a_{i} b_{i} a_{i}^{-1} b_{i}^{-1}$.

The orbit space $D / \Lambda_{g}$ is an orientable surface of genus $g . \Lambda_{g}$ is called an orientable surface group and is a Fuchsian group. The canonical projection $\Pi: D \rightarrow D / \Lambda_{g}$, which defines $D$ as a smooth covering of $D / \Lambda_{g}$, induces an analytic structure on $D / \Lambda_{g}$ in such a way as to make $D / \Lambda_{g}$ into a Riemann surface and $\Pi$ an analytic map.

The second type of group, which we denote by $\Lambda_{p}$, has the presentation

$$
\left\{a_{1}, a_{2}, \ldots a_{p} ; \quad a_{1}^{2} a_{2}^{2} \ldots a_{p}^{2}=1\right\} \quad(p \geqq 3) .
$$

The orbit space $D / \Lambda_{p}$ is a non-orientable surface of genus $p$ (i.e. a sphere with $p$ cross-caps). $\Lambda_{p}$ is called a non-orientable surface group and is a proper NEC group, the $a_{i}$ being glide reflections. The canonical projection $\Pi: D \rightarrow D / \Lambda_{p}$, which again defines $D$ as a smooth covering of $D / \Lambda_{p}$, induces an analytic and anti-analytic structure on $D / \Lambda_{p}$, so that $D / \Lambda_{p}$ becomes a Riemann surface and $\Pi$ an analytic or anti-analytic map.

(We shall reserve the suffix $g$ for orientable surface groups and the suffix $p$ for nonorientable surface groups.) 
From the Gauss-Bonnet theorem we can deduce the following two formulae

$$
\mu\left(\Lambda_{g}\right)=4 \pi(g-1), \quad \mu\left(\Lambda_{p}\right)=2 \pi(p-2) .
$$

Now from (2) we deduce that the genus of the orientable surface $D / \Lambda_{p}^{+}$is $p-1 . D / \Lambda_{p}^{+}$ is the orientable two-sheeted covering surface of $D / \Lambda_{p}$.

If $\Gamma$ is an NEC group, denote the $\Gamma$-orbit of $z$ by $[z]_{\Gamma}$, so that $[z]_{\Gamma} \in D / \Gamma$. We can now write the canonical projection from $D / \Lambda_{p}^{+} \rightarrow D / \Lambda_{p}$ by

$$
[z]_{\Lambda_{p}^{+}} \rightarrow[z]_{\Lambda_{p}},
$$

and hence it is a smooth analytic or anti-analytic map.

Let $S$ be any non-orientable Riemann surface of genus $p \geqq 3$. Then its orientable twosheeted covering surface $S^{+}$has genus $g \geqq 2$ and hence, by uniformization theory, has $D$ as its universal covering space. Thus $D$ is the universal covering space of $S$ and we deduce that there exists a non-orientable surface group $\Lambda_{p}$ such that

$$
S=D / \Lambda_{p} \text {. }
$$

If $f: S \rightarrow S$ is an automorphism of $S$ then by standard techniques, as described for instance in [4], $f$ may be written

$$
f[z]_{\Lambda_{p}} \rightarrow[g z]_{\Lambda_{p}}
$$

where $g \in \mathscr{G}$ and $g \Lambda_{p}=\Lambda_{p} g$.

We deduce that any group of automorphisms of $S$ is of the form $\Gamma / \Lambda_{p}$ where $\Gamma$ is a proper NEC group. Conversely $\Gamma / \Lambda_{p}$ acts as a group of automorphisms of $D / \Lambda_{p}$, because if $g \in \Gamma$ then the mapping

$$
[z]_{\Lambda_{p}} \rightarrow[g z]_{\Lambda_{p}}
$$

is an automorphism and, if we vary $g$ over its $\Lambda_{p}$ coset, it does not alter the automorphism.

The corresponding result for orientable Riemann surfaces is similar and well known. A group $G$ acts as a group of + automorphisms of $D / \Lambda_{g}$ if and only if $G=\Gamma / \Lambda_{g}$ where $\Gamma$ is a Fuchsian group. It is clear that $G$ acts as a group of automorphisms of $D / \Lambda_{g}$, if and only if $G=\Gamma / \Lambda_{g}$, where $\Gamma$ is an NEC group.

The order of the groups $\Gamma / \Lambda_{g}, \Gamma / \Lambda_{p}$ are calculated as follows:

$$
\begin{aligned}
& \left|\Gamma / \Lambda_{\theta}\right|=\frac{\mu\left(\Lambda_{\theta}\right)}{\mu(\Gamma)}=\frac{4 \pi(g-1)}{\mu(\Gamma)}, \\
& \left|\Gamma / \Lambda_{p}\right|=\frac{\mu\left(\Lambda_{p}\right)}{\mu(\Gamma)}=\frac{2 \pi(p-2)}{\mu(\Gamma)} .
\end{aligned}
$$

Thus a group of automorphisms of a non-orientable (orientable) Riemann surface of genus $p \geqq 3(g \geqq 2)$ is finite.

THEOREM 1. A necessary and sufficient condition for a finite group $G$ to be a group of automorphisms of a non-orientable Riemann surface of genus $p \geqq 3$ is that there exists a proper 
$N E C$ group $\Gamma$ and a homomorphism $\theta: \Gamma \rightarrow G$ such that the kernel of $\theta$ is a surface group and $\theta\left(\Gamma^{+}\right)=G$.

Proof. First we suppose that $G$ is a group of automorphisms of a non-orientable Riemann surface of genus $p \geqq 3$. Then, from the above, $G=\Gamma / \Lambda_{p}$, where $\Lambda_{p}$ is a non-orientable surface group and $\Gamma$ is a proper NEC group. Hence there exists a homomorphism $\theta: \Gamma \rightarrow G$ whose kernel is a non-orientable surface group. Thus there exists

$$
t \in \operatorname{ker} \theta \cap\left(\Gamma-\Gamma^{+}\right) \text {. }
$$

Write $\Gamma=\Gamma^{+}+t \Gamma^{+}$and $\theta\left(\Gamma^{+}\right)=G^{+}$. Then

$$
G=\theta(\Gamma)=\theta\left(\Gamma^{+}+t \Gamma^{+}\right)=\theta\left(\Gamma^{+}\right)+\theta(t) \theta\left(\Gamma^{+}\right)=G^{+}+G^{+}=G^{+} .
$$

Thus $\theta\left(\Gamma^{+}\right)=G$.

For the converse, suppose that there exists a homomorphism $\theta: \Gamma \rightarrow G$ such that $\theta\left(\Gamma^{+}\right)=G$ and $\operatorname{ker} \theta$ is a surface group $\Lambda$. Now, if $\Lambda$ were an orientable surface group, $\Lambda \triangleleft \Gamma^{+}$, so that $\Lambda$ is the kernel of the restriction of $\theta$ to $\Gamma^{+}$. Thus

$$
G=\Gamma^{+} / \Lambda \simeq \Gamma / \Lambda
$$

which is evidently impossible. Thus $\Lambda$ is a non-orientable surface group and $G$ is a group of automorphisms of a non-orientable Riemann surface of genus $p \geqq 3$.

COROLlaRY. If $G$ is a group of automorphisms of a non-orientable Riemann surface, then $G$ is a group of +automorphisms of its orientable two-sheeted covering surface.

Proof. By the theorem there exists a proper NEC group $\Gamma$ and a homomorphism $\theta: \Gamma \rightarrow G$ such that $\theta\left(\Gamma^{+}\right)=G$. Let $\theta^{+}$be the restriction of $\theta$ to $\Gamma^{+}$. Then $\theta^{+}: \Gamma^{+} \rightarrow G$ and it is easy to see that

$$
\left(\operatorname{ker} \theta^{+}\right)=(\operatorname{ker} \theta)^{+},
$$

so that $G$ is a group of + automorphisms of $D /(\operatorname{ker} \theta)^{+}$, which is the orientable two-sheeted covering surface of $D / \operatorname{ker} \theta$.

3. Large groups of automorphisms of non-orientable Riemann surfaces. From (5) we see that we obtain large groups of automorphisms by finding finite groups which are homomorphic images of NEC groups with small measure of fundamental region.

The measure of a fundamental region of a Fuchsian group is bounded below by $\pi / 21$ and this bound is attained only for the $(2,3,7)$ group, i.e. the group with presentation

$$
\left\{x, y ; x^{2}=y^{3}=(x y)^{7}=1\right\} \text {. }
$$

This group may be obtained as follows. Consider a non-Euclidean triangle with angles $\pi / 2$, $\pi / 3, \pi / 7$ and let $\Delta$ be the proper NEC group generated by the reflections $c_{1}, c_{2}, c_{3}$ in the three sides of the triangle. $\Delta$ has the presentation

$$
\left\{c_{1}, c_{2}, c_{3} ; c_{1}^{2}=c_{2}^{2}=c_{3}^{2}=\left(c_{1} c_{2}\right)^{2}=\left(c_{2} c_{3}\right)^{3}=\left(c_{1} c_{3}\right)^{7}=1\right\} \text {. }
$$

$\Delta^{+}$is the $(2,3,7)$ group with presentation (6) $\left(x=c_{1} c_{2}, y=c_{2} c_{3}\right)$. 
$\mu(\Delta)=\pi / 42$ and $\Delta$ is the NEC group which has the smallest area of fundamental region. It is easy to see that $\Delta$ is the only group up to isomorphism with this property.

As the measure of fundamental region of a Fuchsian group is bounded below by $\pi / 21$, Hurwitz's result that the order of a group of +automorphisms of an orientable Riemann surface of genus $g$ cannot be bigger than $84(g-1)$ follows by $(5.1)$. Moreover, as $\mu(\Gamma)=\pi / 21$ if and only if $\Gamma \simeq \Delta^{+}$, it follows from (5.1) that $G$ is a group of $84(g-1)+$ automorphisms of a compact orientable Riemann surface of genus $g$, if and only if

$$
G \simeq \Delta^{+} / \Lambda_{g}
$$

where $\Lambda_{g}$ is an orientable surface group. Now the only normal subgroups of $\Delta^{+}$are orientable surface groups. For they are Fuchsian groups, and if they are not surface groups they must possess elements of finite order. It is known that the only elements of finite order in $\Delta^{+}$are conjugate to $x, y$, or $x y$. Thus a normal subgroup $N$ containing elements of finite order must contain $x, y$, or $x y$. Suppose that $x \in N$. Then under the canonical homomorphism from $\Delta^{+}$to $\Delta^{+} / N, x$ must map to $e$, the identity. Suppose that $y$ maps to $\bar{y}$. Then, from (6),

$$
\bar{y}^{3}=(e \bar{y})^{7}=1 \text {, }
$$

so that $\bar{y}=1$ and $N=\Delta^{+}$. Similarly, if $N$ contains $y$ or $x y, N=\Delta^{+}$. It now follows that $G$ is a group of $84(g-1)$ +automorphisms of an orientable Riemann surface of genus $g$ if and only if $G$ is a finite factor group of $\Delta^{+}$; i.e. $G$ is generated by two elements $X, Y$, which obey the relations

$$
X^{2}=Y^{3}=(X Y)^{7}=1 .
$$

This is another result of Hurwitz and we shall call such a finite group $G$ a Hurwitz group.

Now let us consider the non-orientable case. If $\Gamma$ is an NEC group, then $\mu(\Gamma) \geqq \pi / 42$ and $\mu(\Gamma)=\pi / 42$ if and only if $\Gamma \simeq \Delta$. Hence, from (5.2), the order of a group $G$ of automorphisms of a non-orientable surface of genus $p$ is bounded above by $84(p-2)$ and equality holds if and only if

$$
G \simeq \Delta / \Lambda_{p}
$$

where $\Lambda_{p}$ is a non-orientable surface group. Also, by exactly the same methods as before, we can show that all normal subgroups of $\Delta$ of index greater than 2 are surface groups.

DEFINITION. A group of $84(p-2)$ automorphisms of a non-orientable Riemann surface of genus $p$ will be called an $H^{*}$-group.

THEOREM 2. $G$ is an $H^{*}$-group if and only if it is finite and contains three generators $C_{1}, C_{2}, C_{3}$, which obey the relations

$$
C_{1}^{2}=C_{2}^{2}=C_{3}^{2}=\left(C_{1} C_{2}\right)^{2}=\left(C_{2} C_{3}\right)^{3}=\left(C_{1} C_{3}\right)^{7}=1,
$$

and $G$ is generated by $C_{1} C_{2}$ and $C_{2} C_{3}$.

Proof. If $G$ is an $H^{*}$-group, there exists a homomorphism $\theta: \Delta \rightarrow G$ such that $\theta\left(\Delta^{+}\right)=G$, and so $G$ has generators as described in the theorem. Conversely, if $G$ has these generators, 
there exists a homomorphism $\theta: \Delta \rightarrow G$ such that $\theta\left(\Delta^{+}\right)=G$ and the kernel of $\theta$ must be a surface group, as all normal subgroups of $\Delta$ of index greater than 2 are surface groups. By applying Theorem 1 we deduce that $G$ is an $H^{*}$-group.

COROLLARY (a). If $G$ acts as an $H^{*}$-group on a non-orientable surface $S$, then $G$ acts as a Hurwitz group on the orientable two-sheeted covering surface of S. In particular, every $H^{*}$-group is a Hurwitz group.

Proof. This follows from the corollary to Theorem 1.

COROLLARY (b). A Hurwitz group $G$ generated by $X, Y$, which obey the relations (8), is an $H^{*}$-group if and only if there exists $Z \in G$ such that

$$
Z^{2}=(Z X)^{2}=(Z Y)^{2}=1 \text {. }
$$

Proof. If such a $Z$ exists, then $G$ is generated by $C_{1}=Z X, C_{2}=Z, C_{3}=Z Y$ obeying the relations (9) and also $C_{1} C_{2}=X, C_{2} C_{3}=Y$. Thus, by the Theorem, $G$ is an $H^{*}$-group. Conversely, if $G$ is an $H^{*}$-group generated by $C_{1}, C_{2}, C_{3}$ obeying (9) and also generated by $X=C_{1} C_{2}, Y=C_{2} C_{3}$, then $Z=C_{2}$ obeys the relations (10).

4. The existence of $H^{*}$-groups. In Corollary $(a)$ of Theorem 2 we showed that an $H^{*}$-group is a Hurwitz group. Thus, when looking for $H^{*}$-groups, we need only look amongst Hurwitz groups. Macbeath [5] has determined all the linear fractional Hurwitz groups. We state his result:

$\operatorname{PSL}(2, q)$ is a Hurwitz group if and only if (i) $q=p$, where $p$ is prime and $p \equiv \pm 1(\bmod 7)$, or (ii) $q=p^{3}$, where $p$ is prime and $p$ 丰 $0, \pm 1(\bmod 7)$, or (iii) $q=7$.

In case (i) there are three distinct orientable Riemann surfaces upon which the group acts as a Hurwitz group. In cases (ii) and (iii) there is only one such Riemann surface.

The two smallest Hurwitz groups are $\operatorname{PSL}(2,7)$ and $\operatorname{PSL}(2,8)$, which act on surfaces of genus $g=3, g=7$ respectively.

THEOREM 3. (i) $P S L(2,7)$ is not an $H^{*}$-group, (ii) $\operatorname{PSL}(2,8)$ is an $H^{*}$-group.

Thus the smallest value of the genus for which a non-orientable Riemann surface admits $84(p-2)$ automorphisms is $p=8$.

Proof (i). $|\operatorname{PSL}(2,7)|=7 \times 24$ so that $Z_{7}$ is a Sylow 7 -subgroup of $\operatorname{PSL}(2,7)$. By the Sylow theorems there are eight $Z_{7}$ 's and they are all conjugate; therefore the normalizer of a $Z_{7}$ has order $168 / 8=21$. If $P S L(2,7)$ were an $H^{*}$-group, then by the relations (9) we would deduce that $\operatorname{PSL}(2,7)$ contains a dihedral group of order 14 , which in turn contains a $Z_{7}$ normal in the dihedral group. Thus the dihedral group is a subgroup of the normalizer, which is a contradiction because 14 does not divide 21 .

(ii). It has been shown by Macbeath [5] that two elements $X, Y$ of a linear fractional Hurwitz group, which obey the relations (8), generate the group. We thus look for two such elements $X, Y$ in $P S L(2,8)$. It is known that two elements of $P S L(2,8)$ are conjugate if and only if they have the same trace (Newman [6]). Elements of $P S L(2, q)$ of order 2 have trace 0 , elements of order 3 have trace 1 and elements of order 7 have trace $\xi$ where

$$
\xi^{3}+\xi^{2}-2 \xi-1=0 \text {. }
$$

(In $G F\left(2^{3}\right)$ we can write this last equation as $\xi^{3}+\xi^{2}+1=0$.) 
As $X$ has order two we may assume that

$$
X=\left(\begin{array}{ll}
1 & 1 \\
0 & 1
\end{array}\right)
$$

Let

$$
X Y=\left(\begin{array}{ll}
x & y \\
z & w
\end{array}\right)
$$

where $x w-y z=1$, so that

$$
Y=\left(\begin{array}{cc}
x+z & y+w \\
z & w
\end{array}\right)
$$

Thus we want $x, y, z, w$ to satisfy

$$
x+w+z=1, \quad x+w=\xi, \quad x w-y z=1,
$$

and we see that $x=\xi, y=\xi^{2}, z=1+\xi, w=0$ are solutions.

By Corollary $(b)$ of Theorem 2 we need to find $Z \in P S L(2,8)$ such that

$$
Z^{2}=(Z X)^{2}=(Z Y)^{2}=1 \text {. }
$$

Let

$$
Z=\left(\begin{array}{ll}
\alpha & \beta \\
\gamma & \delta
\end{array}\right)
$$

where $\alpha \delta-\beta \gamma=1$. Then

$$
Z X=\left(\begin{array}{ll}
\alpha & \alpha+\beta \\
\gamma & \gamma+\delta
\end{array}\right), \quad Z Y=\left(\begin{array}{ll}
\alpha+\beta(1+\xi) & \alpha \xi^{2} \\
\gamma+\delta(1+\xi) & \gamma \xi^{2}
\end{array}\right) .
$$

As the traces of $Z, Z X, Z Y$ are all zero we have the equations

$$
\alpha+\delta=0, \quad \alpha+\gamma+\delta=0, \quad \alpha+\beta(1+\xi)=0, \quad \alpha \delta-\beta \gamma=1,
$$

and a solution of these equations is $\alpha=1, \beta=\xi^{2}, \gamma=0, \delta=1$. We deduce that

$$
Z=\left(\begin{array}{ll}
1 & \xi^{2} \\
0 & 1
\end{array}\right)
$$

obeys the equations $(10)$ so that $\operatorname{PSL}(2,8)$ is an $H^{*}$-group.

By similar methods we can show that $\operatorname{PSL}(2,13)$ is an $H^{*}$-group, whilst $\operatorname{PSL}(2,27)$ is a Hurwitz group which is not an $H^{*}$-group.

5. Infinite families of groups. We have shown that $H^{*}$-groups exist and that Hurwitz groups exist which are not $H^{*}$-groups. In this paragraph we show that there are infinitely many $H^{*}$-groups. These considerations lead us to find new infinite families of Hurwitz groups. 
Let $\Lambda_{p}$ be a non-orientable surface group such that $D / \Lambda_{p}$ admits an $H^{*}$-group as its automorphism group. This exists, by Theorem 3(ii) and we know that $\Lambda_{p} \triangleleft \Delta$. The commutator subgroup $\left[\Lambda_{p}, \Lambda_{p}\right]$ is a characteristic subgroup and is a Fuchsian group, as every commutator preserves orientation. The subgroup $\Lambda_{p}^{m}$ of $\Lambda_{p}$ generated by the $m$ th powers of elements of $\Lambda_{p}$ is also a characteristic subgroup and is a proper NEC group if and only if $m$ is odd. Thus for odd $m$ the product

$$
M(m)=\Lambda_{p}^{m}\left[\Lambda_{p}, \Lambda_{p}\right]
$$

is a characteristic subgroup of $\Lambda_{p}$ which contains orientation reversing elements. $\Lambda_{p} / M(m)$ is a finitely generated abelian group in which every element has finite order. Hence it is finite, so that $M(m)$ has finite index in $\Lambda_{p}$ and is thus a non-orientable surface group. Also, as $M(m)$ is characteristic in $\Lambda_{p}, M(m)$ is normal in $\Delta$. Thus $D / M(m)$ is a non-orientable Riemann surface of genus $p^{\prime}$ which admits a group of $84\left(p^{\prime}-2\right)$ automorphisms. We now calculate $p^{\prime}$. $\Lambda_{p}$ has the presentation given by (3.2), so that $\Lambda / M(m)$ will have the presentation

$$
\left\{\bar{a}_{1}, \bar{a}_{2}, \ldots \bar{a}_{p} ; \quad m \bar{a}_{1}=m \bar{a}_{2}=\ldots=m \bar{a}_{p}=2\left(\bar{a}_{1}+\bar{a}_{2}+\ldots+\bar{a}_{p}\right)=0\right\},
$$

where $\bar{a}_{l}$ is the image of $a_{i}$ under the canonical homomorphism. As $m\left(\bar{a}_{1}+\bar{a}_{2}+\ldots+\bar{a}_{p}\right)=0$ and $m$ is odd, $\bar{a}_{1}+\bar{a}_{2}+\ldots+\bar{a}_{p}=0$. Hence $\Lambda_{p} / M(m)$ has the presentation

$$
\left\{\bar{a}_{1}, \bar{a}_{2}, \ldots \bar{a}_{p-1} ; \quad m \bar{a}_{1}=m \bar{a}_{2}=\ldots m \bar{a}_{p-1}=0\right\} .
$$

Therefore $\Lambda_{p} / M(m) \simeq Z_{m}^{p-1}$ and so $\left|\Lambda_{p} / M(m)\right|=m^{p-1}$. Hence

$$
\frac{\mu(M(m))}{\mu\left(\Lambda_{p}\right)}=\frac{2 \pi\left(p^{\prime}-2\right)}{2 \pi(p-2)}=m^{p-1},
$$

and thus $p^{\prime}=m^{p-1}(p-2)+2$. This gives us the following result.

THEOREM 4. If there is a non-orientable Riemann surface of genus $p$ which admits a group of $84(p-2)$ automorphisms, then there is a non-orientable Riemann surface of genus $p^{\prime}$, where

$$
p^{\prime}=m^{p-1}(p-2)+2
$$

for every odd positive integer $m$ which admits a group of $84\left(p^{\prime}-2\right)$ automorphisms.

We know that there is a non-orientable Riemann surface of genus 8 which admits a group of 504 automorphisms, so that we can deduce

COROLlARY. For each odd positive integer $m$ there is a non-orientable Riemann surface of genus

$$
p^{\prime}=6 m^{7}+2 \text {, }
$$

which admits a group of $84\left(p^{\prime}-2\right)$ automorphisms.

By combining Corollary (a) of Theorem 2 with Theorem 4 we see that if there is a nonorientable Riemann surface of genus $p$, which admits a group of $84(p-2)$ automorphisms, then there is an orientable Riemann surface of genus $g^{\prime}$, which admits a group of $84\left(g^{\prime}-1\right)$ + automorphisms, where

$$
g^{\prime}=m^{g}(g-1)+1, \quad g=p-1
$$

for each odd positive integer $m$. 
Now consider $m$ to be even. $M(m)$ will be an orientable surface group and $M(m) \triangleleft \Delta$. Hence $M(m) \triangleleft \Delta^{+}$. Thus $D / M(m)$ is an orientable Riemann surface of genus $g^{\prime}$ admitting a group of $84\left(g^{\prime}-1\right)+$ automorphisms. Now, as we saw above, $\Lambda_{p} / M(m)$ has the presentation (11). Put $\bar{a}_{1}+\bar{a}_{2}+\ldots+\bar{a}_{p}=\bar{b}$; then $\Lambda_{p} / M(m)$ has the presentation

$$
\left\{\bar{a}_{1}, \bar{a}_{2} \ldots \bar{a}_{p-1}, b ; \quad m \bar{a}_{1}=m \bar{a}_{2}=\ldots=m \bar{a}_{p-1}=2 \bar{b}=0\right\} .
$$

Thus $\Lambda_{p} / M(m) \simeq Z_{2} \times Z_{m}^{p-1}$ and so $\left|\Lambda_{p} / M(m)\right|=2 m^{p-1}$. Therefore

and so

$$
\frac{\mu(M(m))}{\mu\left(\Lambda_{p}\right)}=\frac{4 \pi\left(g^{\prime}-1\right)}{2 \pi(p-2)}=2 m^{p-1}
$$

$$
g^{\prime}=m^{p-1}(p-2)+1=m^{g}(g-1)+1,
$$

where $g=p-1$ is the genus of the orientable two-sheeted covering surface of $D / \Lambda_{p}$. Noting (12) we deduce the following result.

THEOREM 5. Suppose that we have an orientable Riemann surface of genus $g$ which admits a group of $84(g-1)+$ automorphisms and which is the orientable two-sheeted covering surface of a non-orientable Riemann surface of genus $p=g+1$, which admits a group of $84(p-2)$ automorphisms. Then for every positive integer $m$ there is an orientable Riemann surface of genus

which admits a group of $84\left(g^{\prime}-1\right)+$ automorphisms.

$$
g^{\prime}=m^{g}(g-1)+1
$$

This improves a result of Macbeath [3] and Lehner and Newman [2], who showed that if there is an orientable Riemann surface of genus $g$, which admits a group of $84(g-1)+$ automorphisms, then there is an orientable Riemann surface of genus

$$
g^{\prime}=m^{2 g}(g-1)+1
$$

which admits a group of $84\left(g^{\prime}-1\right)$ + automorphisms. Thus, for example, the infinite family of integers of the form $6 m^{14}+1$ has been extended to the infinite family of integers of the form $6 m^{7}+1$.

THEOREM 6. For infinitely many values of $g$, there exists an orientable Riemann surface of genus $g$ which admits a Hurwitz group as its +automorphism group and which is not the orientable two-sheeted covering surface of a non-orientable Riemann surface of genus $p=g+1$, which admits an $H^{*}$-group as its automorphism group.

Proof. By Theorem 3(i) we know PSL $(2,7)$ is a Hurwitz group, which is not an $H^{*}$-group. $P S L(2,7)$ is a group of 168 + automorphisms of an orientable Riemann surface of genus 3, so that there exists an orientable surface group $\Lambda \triangleleft \Delta^{+}$such that $D / \Lambda$ is this surface. We now claim that $\Lambda \Delta \Delta$. For, if this were not true, there would exist $c \in \Delta-\Delta^{+}$such that $c \Lambda c^{-1}$ is different from $\Lambda$. If $c \Lambda c^{-1}$ were not conjugate to $\Lambda$ in $\mathscr{G}^{+}$, then $D / \Lambda$ and $D / c \Lambda c^{-1}$ would be two distinct Riemann surfaces of genus 3, which admit $\operatorname{PSL}(2,7)$ as their +automorphism group. This would contradict the theorem of Macbeath stated at the beginning of Section 4. If $c \Lambda c^{-1}$ is conjugate to $\Lambda$ in $\mathscr{G}^{+}$, then there exists $g \in \mathscr{G}^{+}$such that $g c \Lambda c^{-1} g^{-1}=\Lambda$. Thus $g c \in N(\Lambda)$, the normalizer of $\Lambda$ in $\mathscr{G}$. But $\Delta^{+} \subset N(\Lambda)$, and so $N(\Lambda)=\Delta$ as this is the only proper NEC group containing $\Delta^{+}$. Thus we have shown that $\Lambda \triangleleft \Delta$. 
Let $\Gamma$ be a characteristic subgroup of finite index in $\Lambda$. An infinite number of these exist, as for each positive integer $m, \Lambda^{m}[\Lambda, \Lambda]$ is one. We now show that for each such subgroup $\Gamma, D / \Gamma$ is a Riemann surface with the required properties. The proof is by contradiction. If $D / \Gamma$ did not have the properties of the theorem, then there would exist a non-orientable surface group $\Gamma^{*}$ such that $\left(\Gamma^{*}\right)^{+}=\Gamma$ and $\Gamma^{*} \triangleleft \Delta$. Write

and hence

$$
\Gamma^{*}=\Gamma+\gamma \Gamma \text {, where } \gamma \in \Gamma^{*}-\Gamma \subset \Delta,
$$

$$
\gamma \Lambda \gamma^{-1}=\Lambda \text { and } \gamma^{2} \in \Gamma \subset \Lambda \text {. }
$$

Let $\Lambda^{*}=\Lambda+\gamma \Lambda$. Then $\Lambda^{*}$ is a proper NEC group and $\left(\Lambda^{*}\right)^{+}=\Lambda$. Let $t \in \Delta$. Then

$$
t \Lambda^{*} t^{-1}=t(\Lambda+\gamma \Lambda) t^{-1}=t \Lambda t^{-1}+t \gamma \Lambda t^{-1}=\Lambda+t \gamma t^{-1} t \Lambda t^{-1}=\Lambda+t \gamma t^{-1} \Lambda \text {. }
$$

But $\Gamma^{*} \Delta \Delta$, and therefore $t \gamma t^{-1} \in \Gamma^{*}=\Gamma+\gamma \Gamma$.

As $t \gamma t^{-1}$ is orientation reversing, there exists $\gamma^{\prime} \in \Gamma \subset \Lambda$ such that $t \gamma t^{-1}=\gamma \gamma^{\prime}$. Thus

$$
t \Lambda^{*} t^{-1}=\Lambda+t \gamma t^{-1} \Lambda=\Lambda+\gamma \gamma^{\prime} \Lambda=\Lambda+\gamma \Lambda=\Lambda^{*} \text {. }
$$

Thus $\Lambda^{*} \triangleleft \Delta$ and hence must be a non-orientable surface group. This, however, would imply that $\Delta / \Lambda^{*} \simeq \operatorname{PSL}(2,7)$ would be an $H^{*}$-group contradicting Theorem 3(i).

A calculation similar to that used in the proof of Theorem 4 shows that, for $g^{\prime}=2 m^{6}+1$, there exists an orientable Riemann surface of genus $g^{\prime}$, which admits $84\left(g^{\prime}-1\right)$ + automorphisms but which is not the orientable two-sheeted covering of a non-orientable Riemann surface admitting $84\left(p^{\prime}-2\right)$ automorphisms $\left(p^{\prime}=g^{\prime}+1\right)$.

I would like to thank Professor A. M. Macbeath for his advice and encouragement. This paper forms part of a Ph.D. thesis which I wrote under his supervision at the University of Birmingham.

\section{REFERENCES}

1. A. Hurwitz, Über algebraische Gebilde mit eindeutigen Transformationen in sich, Math. Ann. 41 (1879), 428-471.

2. J. Lehner and $M$. Newman, On Riemann surfaces with maxinal automorphism groups, Glasgow Math. J. 8 (1967), 102-112.

3. A. M. Macbeath, On a theorem of Hurwitz, Proc. Glasgow Math. Assoc. 5 (1961), 90-96. (1961).

4. A. M. Macbeath, Discontinuous groups and birational transformations, Dundee Summer School 1967).

5. A. M. Macbeath, Generators of the linear fractional groups, Proc. Symp. Pure Math. (Houston,

6. M. Newman, Maximal normal subgroups of the modular group, Proc. Amer. Math. Soc. 19 (1968), 1138-1144.

7. H. C. Wilkie, On non-Euclidean crystallographic groups, Math. Z. 91 (1966), 87-102.

\section{UNIVERSITY OF BIRMINGHAM}

PRESENT AdDRESS:

UNIVERSITY OF SOUTHAMPTON 